\title{
Le rôle des bibliothèques en éducation relative à l'environnement
}

Pascale Félizat

\section{(2) OpenEdition \\ Journals}

Édition électronique

URL : http://journals.openedition.org/ere/5862

DOI : $10.4000 /$ ere. 5862

ISSN : 2561-2271

Éditeur

Centr'ERE

Référence électronique

Pascale Félizat, "Le rôle des bibliothèques en éducation relative à l'environnement », Éducation relative à l'environnement [En ligne], Volume 15 - 2 | 2020, mis en ligne le 15 novembre 2020, consulté le 23 février 2021. URL : http://journals.openedition.org/ere/5862 ; DOI : https://doi.org/10.4000/ere.5862 


\title{
Le rôle des bibliothèques en éducation relative à l'environnement
}

\author{
Pascale Félizat
}

1 En décembre 2018, 57 institutions et organisations de la société éducative québécoise se mettent d'accord sur une Stratégie québécoise d'éducation en matière d'environnement et d'écocitoyenneté ${ }^{1}$. Les défis, visions et pistes d'actions identifiés par cette stratégie concernent les milieux d'enseignement formel mais aussi l'éducation non formelle assumée par les parcs, les musées, les centres d'interprétation, les ONG, les associations, les éco-quartiers, les médias et autres. Les bibliothèques, qui font pourtant partie du secteur de l'éducation non formelle, ne sont pas mentionnées dans le document et les principales revues touchant l'éducation à l'environnement ne semblent pas non plus faire des bibliothèques un objet de recherche. Pourtant plus de 1000 bibliothèques publiques couvrent le territoire du Québec ${ }^{2}$. Au Québec comme ailleurs, et bien avant les musées par exemple, elles sont les institutions culturelles les plus fréquentées, avec un nombre toujours croissant de visites mais aussi d'actions d'animations. Dans le milieu des bibliothèques, le thème de l'environnement est de plus en plus présent ${ }^{3}$ et plusieurs professionnel(le)s, au Québec comme ailleurs dans le monde, perçoivent que les bibliothèques, en tant qu'outil de transformation sociétale, pourraient être davantage engagées en ce sens. Ces cinq dernières années, des récits d'initiatives ou des témoignages de l'adoption de nouvelles directives au sein des associations professionnelles montrent une telle intention de prendre part aux efforts nécessaires. Mais que font réellement les bibliothèques en matière d'éducation à l'environnement en Amérique du Nord et dans le reste du monde ? Y retrouve-t-on une diversité d'approches? Leurs actions présentent-t-elle des spécificités dans le champ des interventions possibles en éducation à l'environnement? Leur impact pourrait-il être amélioré si elles s'appuyaient davantage sur la collaboration avec d'autres acteurs de ce champ d'intervention éducative? Voilà tout un champ de recherche à développer. 
2 Dans une perspective exploratoire, nous présenterons en premier lieu quelques résultats d'une recherche bibliographique visant une première caractérisation de l'intervention des bibliothèques lorsque leurs missions et fonctions propres sont mises au service d'une éducation à l'environnement et à l'écocitoyenneté. En raison du caractère relativement récent du sujet, les textes sélectionnés serviront davantage de témoins représentatifs d'un état de situation encore mal perçu des bibliothécaires euxmêmes et a fortiori de leurs partenaires, plutôt que de briques critiques pour l'analyse. Nous tenterons toutefois d'apporter ensuite un certain éclairage sur le potentiel des bibliothèques et les conditions de mise en œuvre d'un tel potentiel à la lumière de ce que l'on sait des évolutions des bibliothèques au $21^{\mathrm{e}}$ siècle et de certains fondements de l'éducation relative à l'environnement.

\section{Trois regards sur la question}

Repérés dans la littérature en bibliothéconomie de plus en plus abondante sur le thème des bibliothèques vertes et de leur engagement en matière de viabilité ou de développement durable, voici donc d'abord un résumé de trois articles qui ont été choisis pour illustrer comment peuvent se positionner les bibliothèques publiques face à la question de l'éducation relative à l'environnement.

\section{Le projet inspirant d'une bibliothèque comme « fabrique d'apprentissage urbain »}

4 Tim Schumann (2018) présente le cas d'une bibliothèque qui a investi ce domaine tout en faisant évoluer ses pratiques. Il s'agit de la bibliothèque de Bad oldesloe, une ville allemande de 25000 habitants, déjà très engagée en matière de protection de l'environnement et soucieuse d'équité sociale ${ }^{4}$. Sous la menace de coupures budgétaires et pour se donner une nouvelle légitimité auprès des tutelles, des partenaires financiers et des citoyen(e)s, cette bibliothèque a adhéré au mouvement vert tout en repensant ses rôles, ses espaces et ses services, en collaboration avec la population. L'objectif est de fonctionner davantage comme une "fabrique d'apprentissage urbain", une "agence de compétences", un "espace citoyen» et un "espace de rencontre informel ». Un makerspace est mis en place, qui permet à des individus ou des groupes de mettre en œuvre leurs propres projets. Les espaces de la bibliothèque, situés à proximité d'une école, sont offerts en soutien à des projets communautaires. On favorise les rencontres et la cohésion sociale autour de la résolution de problèmes écologiques ou sociaux. On fait de la sensibilisation à l'environnement par le biais de la programmation et des services, le tout en appui à la "Stratégie allemande pour la durabilité » et dans la mouvance des déclarations des associations professionnelles ${ }^{5}$. Mais on tâche aussi d'aller au-delà de la vitrine verte ${ }^{6}$.

5 À titre d'exemple, au cours d'évènements appelés " Moissonne ta ville $»^{7}$ (centrés sur l'agriculture urbaine), les citoyens ont été invités à redéfinir leur bibliothèque En organisant ces événements, la bibliothèque publique a souhaité donner à voir aux élus et à la population l'étendue des formes possibles d'interventions de la bibliothèque et illustrer par des exemples concrets, le lien entre les objectifs des bibliothèques publiques et les questions d'environnement et de viabilité. En outre, elle voulait sensibiliser les gens à de nouvelles formes d'apprentissage ainsi qu'à la nécessité de 
disposer de lieux de rencontre ouverts dans une société de l'information numérique. Différents moyens d'intervention ont été ainsi utilisés : ateliers, expositions de photos, échanges de semences, conférences, concours de la graine qui pousse le plus vite pour les plus jeunes, fabrication d'hôtels à insectes, utilisation des médias et tableau d'information, hébergement dans les locaux d'initiatives locales (par exemple un groupe de partage de nourriture), mise en place de réseaux de contacts, organisation de parcours guidés à la découverte de plantes, organisation de circuits en vélo pour identifier les arbres fruitiers urbains pouvant faire l'objet de récoltes, adhésion à la semaine nationale pour les changements sociaux et écologiques et autres initiatives citoyennes en faveur de l'environnement, appui aux autorités locales et autres organismes de la société civile pour leurs programmes de protection de la nature, soutien à l'apprentissage de techniques de pomiculture etc. À force de persévérance et de créativité, la bibliothèque a pu s'intégrer dans les débats politiques et sociaux, adopter un nouveau rôle auprès de la municipalité et la communauté, expérimenter de nouvelles formes de collaboration, donner une autre utilité aux espaces de la bibliothèque, présenter la bibliothèque comme un lieu d'apprentissage moderne et créatif, améliorer la visibilité de la bibliothèque comme partenaire potentiel, assumer la responsabilité de prendre soin de l'environnement local et soutenir de nouvelles initiatives.

\section{Une exploration des enjeux, limites et possibilités}

6 En 2008, un bibliothécaire français constatait que si plusieurs bibliothécaires étaient sensibles à la question écologique, les bibliothèques dans leur ensemble - comme institutions - ne s'étaient pas encore saisies de la question écologique (Caraco, 2008). Dix ans plus tard, Manon Le Guennec (2018) constate que les bibliothèques publiques françaises sont indéniablement des actrices de la sensibilisation et de l'information sur les questions environnementales. Elles intègrent ces questions à leur bâtiment et fonctionnement, mettent en place des collections dédiées au développement durable, ou plus précisément à l'écologie, et insèrent ces sujets d'actualité dans leurs programmations. Mais tout cela ne va pas sans difficultés et enjeux qui selon cette auteure, seraient de trois ordres: la capacité à travailler en partenariat, à se positionner et à communiquer.

7 Tout d'abord, il y a le constat du manque de compétences du personnel des bibliothèques, par exemple lorsqu'il s'agit de mettre en place une grainothèque, un service largement répandu dans les bibliothèques françaises et ailleurs ${ }^{8}$, mais aussi lorsqu'il s'agit de faire adopter le tri sélectif à toute une équipe. Manon Le Guennec constate aussi que si les bibliothécaires interrogé(e)s se saisissent de ces questions, c'est en partie parce qu'ils ou elles y étaient sensibles à titre personnel ${ }^{9}$, la volonté des tutelles constituant soit un terreau favorable, soit un frein à cet engagement personnel. Elle suppose que la relative discrétion des bibliothécaires français(e)s sur leurs activités pourrait venir $\mathrm{du}$ fait que, perçue comme un sujet politique, la défense de l'environnement pourrait mettre mal à l'aise les professionnel(e)s des bibliothèques soumis(es) au devoir de neutralitéi ${ }^{10}$. On craindrait d'accroître des effets de distinction qui feraient que les personnes déjà intéressées par l'écologie viendraient à la bibliothèque et que les autres, non. 
8 La prise en compte des besoins des membres de la communauté et leur implication avant et pendant la mise en place d'actions est un enjeu important pour éviter l'écueil d'être perçu comme prescriptif, ce qui pourrait être le fait de l'école mais pas des bibliothèques, très attachées par exemple à la défense de la «lecture plaisir ». Avant d'engager leur bibliothèque dans des actions à caractère social, plusieurs s'assurent donc que ces actions répondent bien à des besoins identifiés de la communauté et s'engagent parfois dans une gestion réellement participative.

Enfin, Manon Le Guenec constate que les bibliothèques, même si elles mettent en place de nombreuses actions, communiquent peu sur le sujet. Cette observation viendrait en partie du fait que, contrairement aux associations professionnelles étrangères, les associations professionnelles françaises en bibliothéconomie n'auraient pas encore mis en place de groupe de travail sur le sujet ${ }^{11}$. Même si plusieurs journées d'études ont été organisées ces dernières années, les professionnel(e)s resteraient encore "avides d'expériences concrètes». C'est ainsi qu'en 2019, la Commission advocacy de l'Association des bibliothécaires français $(\mathrm{ABF})$ a mis en place une collecte nationale d'actions en lien avec les objectifs de l'Agenda 2030. Plusieurs centaines d'actions ont été répertoriées dans une base de données en ligne assortie de conseils. On y encourage les bibliothèques à mettre en évidence leur engagement en faveur de l'environnement dans leur stratégie de communication auprès de leurs usagers.

\section{Une initiative structurante}

$10 \mathrm{Au}$ regard de ces enjeux, voyons maintenant comment un petit groupe de bibliothécaires américains se sont organisés pour réfléchir ensemble à une stratégie de mobilisation, ont impliqué leur association locale, la New York Library Association (NYLA) puis l'American Library Association (ALA) et, en l'espace de 5 ans, ont réussi créer un début de mouvement pour que les bibliothèques publiques, scolaires et universitaires américaines s'engagent davantage en matière de développement durable (Aldrich et Bollerman, 2019). C'est sur la base d'outils conceptuels et stratégiques tirés de la gestion entrepreneuriale que ce groupe de bibliothécaires s'est engagé en 2015, dans une démarche de réflexion à ce sujet. Ils ont d'abord adopté une vision commune : « des bibliothèques vivantes, visibles et viables qui travaillent à mettre en place une organisation structurée permettant de clarifier l'état souhaité de la bibliothèque mais aussi celui de la communauté en matière de viabilité, de résilience et de capacité à se régénérer ». Ils ont aussi adopté une représentation en étoile de la dynamique de leur projet, mettant en évidence les 5 parties prenantes de celui-ci : la Terre est l'une de ces parties prenantes au même titre que la communauté, les clients, les investisseurs et les "cocréateurs $~^{12}$. Ils ont reconnu l'importance d'équilibrer les besoins de ces différentes parties prenantes et d'aligner les valeurs et les ressources d'une bibliothèque sur le «droit des communautés locales et mondiales à subsister, rebondir après une perturbation et prospérer en se régénérant ». Dans une deuxième phase, plusieurs outils ont été mis en place par un groupe de travail de la NYLA, dont un programme de certification des bibliothèques durables et un programme de formation d'agents de changement. Les responsables de bibliothèques bénéficient ainsi d'un d'accompagnement professionnel d'un an visant à les aider à travailler avec les leaders de la communauté afin de résoudre un problème social ou écologique du milieu. Dans une dernière phase atteinte en 2019, l'American Library Association a été mobilisée. Celle-ci a formulé une "résolution pour l'adoption de la viabilité comme valeur 
fondamentale de la bibliothéconomie» et le développement de «normes d'accréditation pour garantir que le thème de la viabilité soit un élément inhérent au programme scolaire en bibliothéconomie ». Il s'agit d'aider les bibliothèques à devenir des modèles d'organisations gérées de façon durable, mais aussi de les inciter à jouer un rôle dans le développement de la pensée critique nécessaire à l'exercice de la profession de bibliothécaire comme au développement des communautés.

\section{Commentaires critiques}

11 Si le sujet de l'environnement et de la viabilité fait l'objet de discussions depuis des décennies dans les rencontres professionnelles, on perçoit maintenant dans le milieu des bibliothèques, comme dans les autres milieux, une plus grande urgence à agir : mondialement, les bibliothèques défendent de plus en plus auprès de leurs tutelles, leurs partenaires financiers et leurs publics, un "rôle d'exemples, d'éducateurs et de facilitateurs » en matière de viabilité (IFLA, 2018).

\section{L'évolution de la conception des bibliothèques publiques : un enjeu de communication}

Les trois articles choisis montrent une diversité de positionnements. Cette diversité est le reflet de la situation de transition des bibliothèques aujourd'hui. Avec l'Internet et son accès immédiat à ses masses de documents et d'informations, les bibliothèques ont dû en effet se repositionner, notamment autour du concept de bibliothèque "tiers lieu ». Elles deviennent « espaces citoyens » avec des salles de réunion, des installations artistiques, des salles de classe, des scènes de spectacles, des ateliers de fabrication, des aires de jeux, etc. Toutefois, plusieurs auteurs font mention des difficultés des bibliothèques publiques à communiquer cette évolution en dehors de cercles d'initiés et ceci d'autant plus que la traduction dans les faits de cette nouvelle «bibliothèque citoyenne » reste parfois encore embryonnaire ${ }^{13}$. Dans la première décennie des années 2000 , et au moment même où le Québec, parfois très brillamment ${ }^{14}$, tentait de rattraper son retard historique en matière de lecture publique, de brutales réductions des dépenses publiques jointes bien souvent à des efforts simultanés de ré-informatisation et d'automatisation importants ont plombé le développement de ce milieu professionnel comme de bien d'autres. Actuellement, en dépit de ces coupures budgétaires, de gros projets d'équipements d'un nouveau genre se multiplient à Montréal et dans d'autres régions de la province, avec un risque réel de découplage entre vision et réalité de mise en œuvre ${ }^{15}$. En effet, en dépit de l'évolution des discours depuis une dizaine d'années, la multiplication des consultations citoyennes et les injonctions de plus en plus pressantes des tutelles à sortir des silos, innover et collaborer davantage, on peine encore aujourd'hui à changer de posture à l'intérieur même des équipes ${ }^{16}$. Comme dans le milieu scolaire, une formation continue adéquate fait souvent défaut pour accompagner les équipes vers d'autres types de fonctionnement et de compréhension de leur rôle ${ }^{17}$. Face à ce constat d'immobilisme relatif des institutions, de plus en plus d'organisations concurrentes dans le secteur de l'innovation sociale prennent le relais au Québec ${ }^{18}$. Ce contexte n'est pas, bien sûr, propre au Québec. Ce n'est pas un hasard si David Lankes, un bibliothécaire américain connu internationalement pour ses efforts de renouvellement de la profession, 
s'adresse avant tout aux citoyens et partenaires potentiellement bénéficiaires des bibliothèques et non pas aux bibliothécaires eux-mêmes lorsqu'il écrit Expect More : Demanding Better Libraries For Today's Complex World » en 2014 ${ }^{19}$. Il y rappelle que, comme tout au long des quatre millénaires d'histoire des bibliothèques, le cœur de la bibliothéconomie reste la création de connaissances transformatrice des sociétés et non la simple gestion de collections d'artefacts et de services pour des usagers ${ }^{20}$. Il revoit les facettes fonctionnelles des bibliothèques publiques et insiste sur l'importance de mettre la communauté, ses rêves et ses particularités propres au centre des préoccupations et des actions des bibliothèques, invitant les professionnel(le)s des bibliothèques à "écrire l'histoire de la communauté». Lankes ne le dit pas explicitement, mais on peut penser bien sûr que cette notion de communauté soit étendue à la communauté biotique chère à l'écologiste Aldo Léopold, c'est-à-dire non pas seulement la communauté humaine d'un lieu particulier mais l'ensemble des êtres vivants qui y habitent. Ce sont ces facettes fonctionnelles des bibliothèques utilisées par Lankes que nous allons maintenant utiliser pour préciser rapidement ce que font aujourd'hui les bibliothèques, de même que leur potentiel d'interventions.

\section{Un important potentiel en matière d'éducation à l'environnement}

13 À travers leurs différentes facettes d'interventions, les bibliothèques peuvent sans doute rejoindre différents objectifs d'éducation à l'environnement :

\section{Ressources partagées :}

Collections de documents y compris des documents créés avec ou par la communauté mais aussi, de plus en plus fréquemment, prêts d'objets tels que des instruments de mesure, équipements de plein air, microscopes, lunettes astronomique, kits à expérimenter chez soi, etc. ainsi que grainothèques, toutes choses servant ultimement à la connaissance y compris par les conversations et actions qu'elles suscitent. Il s'agit surtout, pour les citoyen-e-s, de réaliser des économies dans le cadre du système marchand par l'achat collectif et le prêt, mais il est intéressant de constater que par extension, les bibliothèques peuvent aussi organiser des échanges d'objets directement entre citoyens et donc encourager l'économie de partage. Le soutien à la mise en place de micro-bibliothèques dans l'espace public en est un exemple.

\section{Stimulant économique :}

15 Mise en valeur de modèles alternatifs d'échanges de biens et de services en montrant qu'on peut partager, économiser ou réutiliser ${ }^{21}$ des ressources locales, en signalant et mettant en valeur les ressources naturelles (par exemple circuit de récoltes, circuit touristique...) et patrimoniales $d u$ territoire (y compris les données ouvertes et le domaine public), en soutenant de diverses façons l'économie sociale et écoresponsable et l'implication citoyenne.

\section{Centre d'apprentissage :}

Animations, conférences, séances de cinéma, formations (y compris en ligne et hors les murs); Scientifiques, environnementalistes ${ }^{22}$ et artistes en résidence; Visites guidées; "Bibliothèques vivantes " ${ }^{23}$,échanges de compétences entre pairs, repérage et contact avec des experts locaux en fonction des intérêts; Mise à disposition d'outils high tech (makerspace, medialab) ou low tech 
(ruche, jardins partagés, foodlab...) pour l'apprentissage par le faire; mise en contact avec des animaux; Soutien à des projets citoyens complexes, etc.

\section{Filet de protection sociale :}

Soutien aux populations vulnérables, y compris les itinérants, les nouveaux-arrivants, les anciens combattants, les jeunes enfants et les apprenants en alphabétisation: activités telles que les heures $d u$ conte mais aussi ressources gratuites comme des connexions à internet, des postes informatiques, des laissez-passer dans les musées... Contribution à la santé psychique des jeunes et moins jeunes, en offrant des lieux de calme et de sécurité, par la qualité des espaces, par exemple des bâtiments avec des éléments naturels, des vues sur le territoire..., par des enseignements, comme le yoga, la méditation, par l'offre de nouvelles occasions de bouger et d'exercer sa créativité ... Mais aussi soutien à la résilience des communautés en cas de crise (inondations, feux, ouragans, crise sociale...).

\section{Gardiennes du patrimoine culturel :}

Il s'agit bien sûr d'une des grandes forces des bibliothèques, souvent dépositaires mais aussi parfois créatrices de documents et données de tous types, y compris des cartes et données sur le territoire, qui permettent de conserver et de mettre en valeur la mémoire de l'histoire de la communauté. Cette communauté peut aussi être la communauté biotique, une avenue à développer ${ }^{24}$. Ces dernières années, à l'instar des galeries, musées et archives, les bibliothèques encouragent de plus en plus souvent la contribution aux communs de la connaissance Wikipédia, Wikidata, Wikisource, etc.

\section{Tiers lieu :}

19 Un espace public qui permet le développement des liens sociaux, la confiance et la cohésion sociale. Ce lieu peut notamment donner à voir des réalisations artistiques sur le thème y compris par les citoyens et citoyennes, par exemple une exposition de photos. Il peut abriter aussi des célébrations des avancées citoyennes et des personnalités phares de la communauté.

\section{Berceau de la démocratie ${ }^{25}$ :}

Dispositifs pour faciliter l'expression, la prise de parole (comme le médialab), le débat et la contribution citoyenne: club de lecture, université autrement, café philo, collection coup de poing invitant à la réflexion critique. Formations juridiques, éducation aux médias et à la recherche d'information, promotion de ressources permettant de combattre les fausses nouvelles, mais aussi et surtout, fourniture d'une information fiable sur les questions environnementales. Quelques bibliothèques ont aussi mis en place des sites ou des pages Facebook spécialisées sur le thème de l'environnement.

\section{Symbole des aspirations de la communauté :}

21 Le bâtiment peut être exemplaire du point de vue de la durabilité et être lui-même source d'apprentissage et d'inspiration ${ }^{26}$. Il peut aussi offrir des équipements comme un point de recyclage de piles, de lunettes ou autres objets, des supports à vélo, des places de stationnement pour le covoiturage et des stations de recharge pour les véhicules électriques. Idéalement, ce lieu reflète aussi la culture et les particularités du territoire. Quelques bibliothèques (par exemple en 
Suède ou au Brésil) mettent également en place des vitrines et autres " coins verts » permanents de démonstration interactive pour attirer l'attention sur les éco-gestes et les innovations en ce domaine, mais aussi pour témoigner des efforts des habitants en matière de durabilité

\section{Un enjeu : des ambiguïtés dans le positionnement}

À travers les trois articles sélectionnés, on voit que le pôle américain représenté par l'association ALA de même que la sphère internationale de bibliothéconomie représentée par l'International Federation of Library Associations and Institutions (IFLA) et depuis 2008, par son groupe ENSULIB s'engagent sans surprise dans la mouvance rassembleuse du développement durable de l'Agenda 2030 des Nations Unies, avec les enjeux importants de détournement ou d'extinction des possibles que cela pose (tel qu'explicités par Sauvé, 201127). Un seul article repéré dans notre recension d'écrits, celui de Civallero (2017), aborde directement la question de la décroissance et de son impact sur les bibliothèques. Lorsqu'on parle des inéluctables bouleversements climatiques, on parle donc surtout ouvertement de s'y préparer (comme dans Aldrich et Bollerman, 2019), mais plus rarement d'accompagner les transformations socio-culturelles profondes qui seraient nécessaires pour en prévenir ou en atténuer les effets et impacts. Pourtant, dans les faits, et comme en témoigne la popularité de l'implantation de grainothèques dans les bibliothèques, c'est bien une toute autre forme d'économie et de relations entre citoyens qui est soutenue de plus en plus par les bibliothèques, sans avoir toujours bien conscience, semble-t-il, de ses possibles soubassements théoriques ${ }^{28}$.

Ainsi, une certaine ambiguïté de positionnement peut se lire dans l'exemple américain rapporté par Aldrich et Bollerman (2019). L'utilisation d'un cadre de référence issu du milieu de l'entreprise comme fondement de la stratégie de durabilité mise en place pour les bibliothèques est pour le moins surprenante: entre autres, la Terre y est considérée comme une "partie prenante ». Ce choix est sans doute surtout révélateur du souci de continuer à parler le langage des investisseurs privés dont dépend largement le sort des bibliothèques américaines. Certes, dans plusieurs de ses écrits ${ }^{29}$, Smith Aldrich semble endosser la vision de Lankes (2018) pour lequel il importe de considérer le public des bibliothèques comme des membres de la communauté ou des voisins et, aussi et surtout, comme des apprenants en recherche de sens et de pouvoir plutôt que comme des usagers et des consommateurs de services. Toutefois il semble que les actions concrètes mises en place aux États-Unis dans la foulée du programme décrit par Aldrich et Bollerman pour l'État de New-York n'aient pas l'envergure pour soutenir une telle vision: un club de lecture à l'échelle de l'état sur l'ouvrage «Drawdown: the most comprehensive plan ever proposed to reverse global warming " (Hawken, 2017), un événement annuel visant à encourager les gens à donner des biens, nettoyer l'environnement, faire des gestes de gentillesse au hasard, etc. ${ }^{30}$, et des programmes pour faire en sorte que les bibliothèques soient des refuges de première ligne en cas de sinistre. En dépit de la volonté affichée au niveau national de collaborer avec des organismes de terrain (Tanner et coll, 2019), il semble qu'on organise tout ce qui peut l'être sans trop se préoccuper de prendre en compte les projets endogènes et contextualisés des habitants de chacun des territoires concernés, qui apparaissent ainsi indifférenciés et sans histoire. 
24 L'exemple allemand de Bad Oldesloe rapporté par Tim Schumann (2018) semble se construire sur des bases sensiblement différentes, avec une réflexion sur les nouvelles façons d'apprendre et la tentative d'inviter la population à les expérimenter. Dans ce cas, l'évolution progressive de l'offre de services se fait main dans la main avec la population et à partir d'elle, ce qui n'est pas aussi clair dans l'exemple américain. Quant à la synthèse de Manon Le Guennec concernant ce qui se passe en France, elle montre aussi les difficultés des professionnels à se positionner sur les questions de l'éducation à l'environnement ${ }^{31}$. L'auteure mentionne la crainte qu'une plus forte implication sur le thème de l'écologie soit perçue par la population comme trop prescriptive, politiquement trop engagée, voire même socialement clivante.

\section{Des paradigmes éducationnels à clarifier et un ancrage nécessaire dans le territoire}

Si le rôle éducationnel des bibliothèques semble revendiqué par les auteurs consultés, il ne semble donc pas y avoir d'attention particulière portée à deux points de vigilance pointés par la recherche en éducation relative à l'environnement: 1) la nécessaire conscience du paradigme éducationnel dans lequel s'exerce ce rôle ${ }^{32}$ et 2) l'importance d'inscrire ses intentions éducatives dans les particularités du territoire et de la communauté qui y habite.

Du point de vue des paradigmes éducationnels (tels qu'explicités par Bertrand et Valois, 1999), plusieurs semblent cohabiter dans le milieu des bibliothèques. Comme organisations, et sans même parler de la façon dont s'y organise le travail d'équipe, elles sont très majoritairement imprégnées du paradigme rationnel et technologique. Par exemple, on parle de formation tout au long de la vie pour s'adapter au marché du travail et encore très souvent, de clients ou d'usagers à qui on offre des services. Lorsqu'on fait valoir que l'on tient à l'autonomie des lecteurs et à la lecture plaisir (par rapport à la lecture obligatoire du système éducatif), c'est le paradigme humaniste et ses valeurs de liberté et de projet personnel qui semble invoqué. Lorsqu'on parle du rôle social des bibliothèques et qu'on les considère comme berceau de la démocratie, ça serait plutôt le paradigme socio-interactionnel qui le serait. Enfin, lorsqu'on met en place un makerspace et des communautés d'apprentissage, comme à Bad oldesloe, une bibliothèque primée deux fois par ENSULIB pour son engagement en matière d'environnement, on retrouve certaines des caractéristiques du paradigme inventif comme la promotion de l'engagement dans les milieux de vie et le développement de la capacité d'invention sociale et de création de nouvelles organisations chez les personnes et les communautés.

Du point de vue du nécessaire ancrage dans la réalité du territoire et de la communauté elle-même, on observe aussi une diversité de pratiques. Si l'importance du partenariat avec des acteurs locaux est défendue mondialement, sa mise en œuvre se fait à des degrés bien divers dans la réalité. Dans le monde des bibliothèques, on parle de « hors les murs» (outreach), "d'agents de liaison», de consultation et, de plus en plus, de démarches de conception collaboratives pour les bâtiments et services ${ }^{33}$. Cependant l'utilisation d'outils d'appropriation du territoire en tant que tel, comme ceux préconisés il y a plus de 10 ans par la Trousse d'outils pour des bibliothèques à l'écoute de la communauté, un programme de recherche-action canadien reconnu internationalement ${ }^{34}$, reste limitée. 
Il est par ailleurs intéressant de constater, qu'en dépit de sa volonté affichée de faire appel à la notion "d'impact collectif» surtout comprise semble-t-il comme une injonction à collaborer davantage en contexte de financement raréfié35, et en dépit aussi de son habituel réflexe d'appuyer ses programmes nationaux sur de la recherche ${ }^{36}$, l'association des bibliothécaires américains ne semble pas avoir recherché dans ce cas une concertation avec des organismes nationaux spécialisés en éducation relative à l'environnement. Les bibliothèques relevant, au Québec comme en France, du Ministère de la Culture et non de l'éducation ${ }^{37}$, il n'est par ailleurs pas étonnant de constater leur difficulté à exister au sein d'une stratégie globale telle que la stratégie lancée par Centr'ERE en 2018 (voir la note 1).

Au-delà de l'enjeu très concret d'arriver à sortir des moules de fonctionnement habituel, il est bien possible que la situation constatée dénote également la difficulté à s'approprier jusqu'au bout les notions de problèmes systémiques et de complexité bien présentes dans la mouvance socio-écologique actuelle et indispensables à la planification d'actions portant ultimement sur des transformations profondes.

\section{Conclusion}

Il est clair que les bibliothèques publiques peuvent jouer un rôle important auprès de la population en matière d'environnement. Ce sont les personnes au sein des bibliothèques qui peuvent faire bouger les choses et plusieurs sont déjà à l'œuvre sur le terrain, montrant ainsi que les bibliothèques peuvent faire une différence auprès de leur communauté. Toutefois, en l'absence d'une réflexion plus approfondie sur leur mission et fonctions, mais aussi en l'absence de considération de leurs angles morts (ambiguïté des paradigmes éducationnels sous-jacents, difficulté à se relier au territoire notamment), ces professionnel(le)s risquent fort, comme le craint Aldrich (2018), de s'en tenir à des initiatives vertes isolées et superficielles qui n'auront pas d'impact (ou trop peu) sur leur communauté. Il faudrait certes creuser davantage le sujet pour mieux saisir la réalité en question, mais il semble que les bibliothécaires publics soient plutôt mal équipés aujourd'hui pour mener des actions d'éducation relative à l'environnement efficaces. Plutôt que de s'employer à maîtriser d'entrée de jeu une telle compétence, une voie plus accessible en ces temps d'urgence est peut-être celle de mieux expliquer le potentiel et les forces des bibliothèques aux experts du domaine de l'éducation relative à l'environnement, aux citoyens et aux organismes déjà engagés sur le terrain, afin que ceux-ci expriment en retour leurs besoins particuliers, territoire par territoire. Aider ces acteurs à rayonner, en mettant à leur disposition l'infrastructure des bibliothèques, accompagner les échanges et l'engagement au cœur des projets citoyens, faciliter la recherche d'une information utile parfois difficilement accessible, fournir un lieu sécuritaire et bienveillant, et offrir des ressources utiles à l'apprentissage en contexte et à l'action collective seraient quelques-unes des pistes prioritaires à explorer. 


\section{BIBLIOGRAPHIE}

Aldrich, R. S. (2018). Resilience (Library Futures Series, Book 2) (Vol. 2). Chicago: American Library Association.

Aldrich, R. S. et Bollerman, M. (2019). All together... How? Building capacity and commitment for change. Communication présentée en juin 2019 au Congrès IFLA WLIC, Athènes. Récupéré le 3 février 2020 de http://library.ifla.org/2453/1/156-aldrich-en.pdf

Audunson, R., Aabø, S., Blomgren, R., Evjen, S., Jochumsen, H., Larsen, H. et Koizumi, M. (2019). Public libraries as an infrastructure for a sustainable public sphere. Journal of Documentation, 75(4), 773-790.

Bertrand, Y. et Valois, P. (1999). Fondements éducatifs pour une nouvelle société. Montréal : Les Éditions Nouvelles, 76-77 et 82-87

Campbell B., De Faveri A., O'Donnell J., Singh s. et Smith E., (2009). Trousse d'outils pour des bibliothèques à l'écoute de la communauté, Working Together Toolkit. Récupéré le 16 février 2020 de http://bv.cdeacf.ca/RA_PDF/140109.pdf

Caraco, A. (2008). Les bibliothèques à l'heure du développement durable. Bulletin des bibliothèques de France (BBF), t. 53, $\mathrm{n}^{\circ}$ 3, p. 75-83. Récupéré le 16 février 2020 de http://bbf.enssib.fr/consulter/ bbf-2008-03-0075-010

Civallero, E. (2017). Bibliothèques et décroissance. Documentation Et Bibliothèques, 63(3), 24-35.

Derbas Thibodeau, F. R. et Poirier, C. (2019). Bibliothèques publiques et virage citoyen : enjeux institutionnels et communicationnels. Communiquer : revue de communication sociale et publique, 26, 47-66. Récupéré le 12 février 2020 de www.erudit.org/en/journals/communiquer/2019-n26communiquer04934/1065379ar.pdf

Hawken, P. (2018). Drawdown: the most comprehensive plan ever proposed to reverse global warming. New York: Penguin books

IFLA (2018). Exemplars, Educators, Enablers: Libraries and Sustainability, How libraries contribute to sustainability. Communication du groupe ENSULIB, 25 September 2018. Récupéré le 1er mars 2020 de www.ifla.org/files/assets/hq/topics/libraries-development/documents/

libraries_and_sustainability.pdf

Lankes, D. (2018). Exigeons de meilleures bibliothèques [ressource électronique] : Plaidoyer pour une bibliothéconomie nouvelle. Les Ateliers de [Sens public]. Récupéré de http://ateliers.senspublic.org/exigeons-de-meilleures-bibliotheques/index.html

Le Guennec, M. (2018). Bibliothèques et écologie: Les bibliothèques de lecture publique françaises et les enjeux environnementaux. Mémoire inédit Diplôme de conservatrice de bibliothèque.

Villeurbanne : École nationale supérieure des sciences de l'information et des bibliothèques. Récupéré le 15 février 2020 de www.enssib.fr/bibliotheque-numerique/documents/68275bibliotheques-et-ecologie-les-bibliotheques-de-lecture-publique-francaises-et-les-enjeuxenvironnementaux.pdf

Martel, M. D., Gauthier, P. et Félizat-Chartier, P. (2019). Les démarches de conception collaborative en bibliothèque. Bulletin des bibliothèques de France (17), pp. 126-135. Récupère de : http://bbf.enssib.fr/matieres-a-penser/les-demarches-de-conception-collaborative-enbibliotheque_68974 
Martin, L. (2015). Valeurs professionnelles et cultures politiques des bibliothécaires en France : continuités ou ruptures générationnelles? Mémoire inédit Diplôme de conservatrice de bibliothèque. Villeurbanne : École nationale supérieure des sciences de l'information et des bibliothèques. Récupéré le 1er février 2020 de www.enssib.fr/bibliotheque-numerique/notices/65177-valeursprofessionnelles-et-cultures-politiques-des-bibliothecaires-en-france-continuites-ou-rupturesgenerationnelles

Meschede, C. et Henkel, M. (2019). Library and information science and sustainable development: a structured literature review. Journal of Documentation, 75(6), 1356-1368.

Peekhaus, W. (2018). Seed Libraries: Sowing the Seeds for Community and Public Library Resilience. The Library Quarterly, 88 (3), 271-285.

Sauvé, L. (2011). La prescription du développement durable en éducation : la troublante histoire d'une invasion barbare. Dans Bader, B. et Sauvé, L. (2011), Éducation, environnement et développement durable : vers une écocitoyenneté critique (p. 17-43). Québec: Les Presses de l'Université Laval, collection « L'espace public ».

Schumann, T. (2018). Urban gardening, foodsharing and makerspaces, best practice in the Stadtbibliothek of Bad Oldesloe, Germany. Dans Hauke, P., Charney, M. et Sahavirta H., Going green: implementing sustainable strategies in libraries around the world: buildings, management, programmes and services (p. 122-134). Berlin / Munich : Editor De Gruyter Saur, IFLA Publications Series 177.

Tanner, R., Aldrich, R. S., Antonelli, M. et Ho, A. K. (2019). Libraries as Sustainability Leaders : ALA's Special Task Force. Portal: Libraries and the Academy, 19(3), 365-374.

Townsend, A.K. (2014). Environmental sustainability and libraries: Facilitating user awareness. Library Hi Tech News, 31(9), 21-23.

\section{NOTES}

1. www.coalition-education-environnement-ecocitoyennete.org/wp-content/uploads/2019/07/ Strategie-Edition-complete.pdf

2. Le site de l'Association des bibliothèques publiques du Québec donne le chiffre de 1051 bibliothèques publiques pour 2017. Toutefois ce chiffre fourni par le Ministère de la Culture pourrait bien être sous-évalué. Une collègue bibliothécaire spécialiste en statistiques aurait dénombré 1400 bibliothèques publiques au Québec auxquelles s'ajoutent bien sûr les nombreuses autres bibliothèques présentes en milieu scolaire et universitaire.

3. Meschede et Henkel, 2019. 295 articles provenant de 102 sources ont été analysés dans cette revue de littérature. Des sujets comme «bibliothèques vertes» ou «technologies de l'information vertes » semblent bien établis. L'étude appelle à poursuivre les efforts de recherche en indiquant quelques pistes: par exemple, la formation de l'opinion sur le thème «bibliothéconomie et développement durable » et la diffusion d'informations sur la viabilité (par exemple, via les médias sociaux ou les systèmes d'information). Il est à noter que, mondialement et comme dans d'autres domaines, le sujet commence à être considéré comme devant faire partie à part entière de l'enseignement et de la recherche en bibliothéconomie. L'ENSSIB, l'école de bibliothéconomie française, a par exemple confié cette année à l'une de ses chercheuresenseignantes une mission de recherche-formation sur le thème bibliothèques et information à l'environnement. À Montréal, depuis 2018, l'École de bibliothéconomie et des sciences de l'information (EBSI) de l'U.de M. sensibilise les étudiant-e-s à ces questions.

4. La ville adhère par exemple au mouvement des villes équitables. 
5. Déclaration de l'IFLA, de ALA et Agenda 2030 pour le développement durable.

6. Schumann (2018), p. 125 : «Le mouvement des bibliothèques vertes est critiqué pour mettre trop d'emphase sur l'architecture et les techniques », «La durabilité ne peut fonctionner que dans le cadre d'une approche holistique et les aspects écologiques doivent être liés aux aspects sociaux, culturels et économiques » (ma traduction).

7. «Harvest your city». Les éditions 2015, 2016 et 2017 sont décrites dans l'article et leurs retombées pour la communauté et la bibliothèque analysées : 2015 est organisé sur le thème des jardins urbains et 2016, sur le thème de la nourriture et de la nature en ville. L'édition 2017 a duré toute une semaine et s'est étendu à toute la ville.

8. La gestion de grainothèques, un commun proche de celui des livres, avec quelques fois échange de plants et mise en place de cultures dans ou à proximité de la bibliothèque est un phénomène international pour les bibliothèques au point d'avoir fait récemment l'objet d'un article de synthèse (Peekhaus W., 2018). La présence de grainothèque est un marqueur du passage à des bibliothèques davantage centrées sur les gens et la communauté. Elle a un impact sur la sécurité alimentaire, la durabilité, l'engagement citoyen et l'éducation communautaire. A travers les grainothèques, les bibliothèques donnent à voir leur capacité à offrir une infrastructure pour la préservation et le partage de savoirs locaux issus de l'expérience. Ces connaissances, les ressources que constitue la collection de graines et la communauté de jardiniers qui en prend soin ont le pouvoir de contribuer à transformer la vie des membres de toute la communauté mais aussi d'accroître la biodiversité et la résilience d'un territoire.

9. Manon Le Guennec signale que l'engagement des bibliothécaires dans des partis politiques ou des associations liées aux questions de société est un phénomène important dans la profession. Elle cite une enquête de Leslie Martin (2015) qui estime la proportion de professionnel(le)s ainsi engagé(e)s à $40 \%$.

10. La question du devoir de neutralité, souvent débattue dans la profession, serait plutôt un devoir d'impartialité d'après Smith Aldrich (Résilience, 2018) et un engagement à défendre l'équité d'accès. Comme nous le rappelle, Lankes, les bibliothèques, historiquement, n'ont jamais été neutres et, du fait de leur mission propre de transformation des sociétés par la création de nouvelles connaissances, ne peuvent qu'être engagées donc non neutres.

11. L'auteure cite la «Task force on environment » aux États-Unis et le «Sustainable Libraries Group " en Australie. Un lieu de discussion existe à l'échelon international sous la forme d'un Special interest group (SIG) de l'IFLA, depuis 2008 (ENSULIB). Quelques bibliothécaires français y participent. Les missions de ce groupe portent sur « les effets du changement climatique sur les bibliothèques, la mise en place des éco-gestes dans les bibliothèques, les recommandations pour la profession, la promotion et le développement des ressources et services en bibliothèque concernant le développement durable et le fait de susciter la conscience environnementale des bibliothécaires ». Ce groupe a organisé en 2017, pour la première fois, une rencontre à Berlin. Il est à noter par ailleurs que le 25 septembre 2020, une rencontre en ligne organisée par Raphaëlle Bats (ENSSIB) sur le thème " bibliothèque et médiation à la transition écologique et solidaire » $a$ rassemblé plus d'une centaine de bibliothécaires francophones.

12. On comprend ici que les cocréateurs sont les membres du groupe de réflexion qui veulent voir arriver ces changements.

13. Voir par exemple, Derbas Thibodeau, F. et Poirier, C. (2019) qui font ce constat à propos de la bibliothèque Marc Favreau à Montréal. L'ouverture de cette bibliothèque publique en 2013, après la Grande bibliothèque en 2005, a été un marqueur important du virage des bibliothèques publiques québécoises vers des milieux de vie d'un nouveau modèle.

14. Les Réseaux BIBLIOS du Québec, des structures de soutien gérées comme des OBNL, ont notamment réussi en quelques décennies à peine, en maximisant la coopération intra et inter régions, à instaurer partout sur le territoire des services de qualité quasi identique aux zones urbaines pour les villes de moins de 5000 habitants. Ce modèle de développement coopératif 
ultra-efficace des bibliothèques rurales québécoises est reconnu à l'international, même s'il est peu célébré des québécois eux-mêmes.

15. Dix projets de bibliothèques mixtes d'un nouveau genre (à la fois centre culturel, communautaire et bibliothèque) sont en cours de développement à Montréal et d'autres gros projets semblables sont prévus à Laval, Gatineau, Québec et ailleurs. Au Québec comme ailleurs, les instances publiques soutiennent les dépenses d'investissement, mais plus difficilement les dépenses de fonctionnement.

16. Près de 14000 personnes travaillent dans les bibliothèques au Québec dont environ 500 bibliothécaires. Les femmes sont majoritaires.

17. Des changements concernant la formation initiale sont toutefois en cours. Par exemple au Québec : https://martel.ebsi.umontreal.ca/

18. Notamment en ce qui concerne les plateformes d'échange de connaissances entre citoyens et autres espaces citoyens avec des initiatives comme le Réseau Accorderie, l'espace public Temps libre Mile-End, le Lab transition du collectif Solon, la plateforme d'échanges E180 ou la plateforme Passerelles du TIESS et bien autres initiatives semblables, particulièrement foisonnantes, du milieu de l'innovation sociale au Québec. Au Québec, et dans les grandes villes au moins, les bibliothèques sont en effet loin d'être les seuls « tiers lieux » mis à la disposition des québécois(e)s. Divers organismes à visée sociale tels que ceux cités plus haut en offrent; Les espaces de cafés et de restauration permettant les rencontres et la discussion se multiplient aussi. Toutefois, les tiers-lieux bibliothèques peuvent potentiellement s'en démarquer par le critère « ouvert gratuitement à tous ».

19. Ce livre a été traduit en français en 2018 par un groupe de bibliothécaires québécois sous le titre « Exigeons de meilleures bibliothèques".

20. La mission d'une bibliothèque est d'améliorer la société en facilitant la création de connaissances dans sa communauté».

21. Les ateliers de réparation sont par exemple en forte augmentation dans les bibliothèques.

22. Le réseau des bibliothèques publiques de Toronto a récemment hébergé une environnementaliste en résidence (Janelle Richards, 2018)

23. Par exemple une rencontre avec un membre des communautés autochtones.

24. Une bibliothécaire de la bibliothèque départementale du Bas-Rhin en France suggérait lors d'une discussion récente entre professionnels: «Pourquoi pas des coups de cœurs sur des espèces vivantes, plutôt que sur des écrivains morts?»

25. Le rôle démocratique des bibliothèques est actuellement débattu dans la littérature. Voir par exemple, Audunson et coll. 2019.

26. Voir par exemple Townsend, A.K., 2014, pour des exemples au Canada.

27. «Le développement durable est un cadre de référence, un moule à penser, beaucoup trop étroit (voire déformant) pour en faire un projet de société et encore moins, un projet éducatif » (Sauvé, 2011).

28. Le travail sur les communs d'Elinor Ostrom, qui a pourtant collaboré avec une bibliothécaire, Charlotte Hess, pour écrire en 2007 «Understanding knowledge as a commons: from theory to practice " et l'importance croissante du mouvement des communs sont encore peu connus de la majorité des bibliothécaires même s'il existe plusieurs pôles militants à cet égard (par exemple à Rennes en France et à Montréal). Cette situation change toutefois progressivement notamment avec l'appropriation croissante de l'outil Wikipédia par la profession.

29. Smith Aldrich, R. (2018) par exemple.

30. The great give back. Cet événement, qui existe depuis 2017, a rassemblé le public de 160 bibliothèques dans l'État de New-York en 2019.

31. La situation des bibliothèques publiques québécoises resterait à analyser. Au niveau mondial, il y a sans doute d'autres variantes dans les approches qu'il serait intéressant de continuer à explorer. On pense notamment à l'Afrique et aux pays d'Amérique centrale et du Sud. 
32. La tentative des bibliothécaires américains de penser les «croyances, philosophies et principes » (Aldrich, R. S., Bollerman, M. (2019), attachées à leur volonté de s'engager davantage en développement durable, avortée de mon point de vue, me semble témoigner de la difficulté à s'engager dans ce type de réflexion.

33. Voir par exemple Martel, M. D., Gauthier, P., et Félizat-Chartier, P., (2019)

34. Campbell B. et coll. (2009). On pense notamment aux outils « entrée dans la communauté » et « cartographie de la communauté ».

35. Aldrich et ses collaborateurs mentionnent que la notion d'« Impact collectif » sera utilisée dans les programmes de formation mis en œuvre. Cette notion est ainsi définie par le Center for the Future of Libraries de l'American Library Association : "Face à des ressources limitées et à des problèmes sociaux importants et persistants (faim, pauvreté, violence, éducation, santé, sécurité publique, environnement), les organisations de différents secteurs adoptent des programmes communs pour lutter contre les problèmes au sein de leurs communautés". Voir www.ala.org/ tools/future/trends/collectiveimpact

36. On pense notamment au programme de littératie familiale «Every Child ready to read » ou encore au travail important engagé autour de l'amélioration des services aux adolescents qui font tous deux l'objet d'importants programmes de recherche aux États-Unis.

37. Même s'il existe quelques cas de bibliothèques mixtes (municipales et scolaires) et que la très grande majorité des bibliothèques publiques reçoivent régulièrement des visites de classes et offre de l'aide aux devoirs, la collaboration entre les deux milieux reste délicate. Pourtant, c'est bien le modèle américain qui inspire les bibliothèques publiques au Québec et ailleurs, et celui-ci prend ses racines au XIXe siècle dans un mouvement des bibliothèques publiques étroitement associé au mouvement d'éducation publique. Melvil Dewey, un père fondateur des bibliothèques modernes, insistait même sur le fait que les bibliothèques publiques sont des établissements d'enseignement au même titre que l'école publique.

\section{RÉSUMÉS}

Que font les institutions bibliothèques en matière d'éducation relative à l'environnement ? Cet article offre une première caractérisation sur la base de trois exemples de mobilisation des bibliothécaires en Allemagne, en France et en Amérique du Nord. Ces exemples témoignent d'une intensification des actions mais aussi d'un certain flottement dans le positionnement. Si l'ensemble des nombreuses facettes fonctionnelles des bibliothèques peuvent être investies, le milieu de la bibliothéconomie bénéficierait sans doute des éclairages de la recherche en éducation relative à l'environnement et à l'écocitoyenneté pour clarifier ses intentions, mieux se coordonner aux autres acteurs de terrain et jouer ainsi un rôle plus significatif dans les évolutions culturelles en cours.

What are library institutions doing in regard to environmental education? This article attempts a first characterization on the basis of three examples of mobilization of librarians in Germany, France and North America. These examples show an intensification of actions but also a certain hesitation in positioning. If all of the many functional facets of libraries can contribute, at this stage, the library community would likely benefit from the insights of research in environmental education and eco-citizenship in order to clarify its intentions, better coordinate with other actors in the field, and thus play a more significant role in the ongoing cultural changes. 


\section{INDEX}

Mots-clés : bibliothèque, éducation relative à l'environnement, culture, transformation des institutions

Keywords : library, environmental education, culture, institutional transformation

\section{AUTEUR}

\section{PASCALE FÉLIZAT}

Pascale Félizat, a travaillé pendant plus de 20 ans comme bibliothécaire gestionnaire et conseillère professionnelle et technique auprès d'équipes de bibliothèques en Guadeloupe, en France et au Québec. Elle se forme actuellement en éducation relative à l'environnement et à l'écocitoyenneté pour tenter d'augmenter la pertinence et la portée des actions engagées dans les bibliothèques de tous types (publiques, scolaires, universitaires...) en facilitant leurs conversations avec les citoyenn-e-s et organismes déjà engagés en ERE sur leur territoire. Son travail est accessible sur la plateforme libre de Territoires innovants en économie sociales et solidaires : le Collaboratoire des bibliothèques en transition. 